\title{
Evaluating Comprehension and Utilization of Variability Aspects in UML-Based Models
}

\author{
Iris Reinhartz-Berger ${ }^{1}$, Arnon Sturm ${ }^{2}$, and Arava Tsoury ${ }^{1}$ \\ ${ }^{1}$ Department of Information Systems, \\ University of Haifa, Haifa 31905, Israel \\ iris@is.haifa.ac.il, aravabt@gmail.com \\ ${ }^{2}$ Department of Information Systems Engineering, \\ Ben-Gurion University of the Negev, Beer Sheva 84105, Israel \\ sturmabgu.ac.il
}

\begin{abstract}
In Software Product Line Engineering (SPLE), the ability of a software artifact to be used in different contexts is very essential for productivity. In order to manage and support this ability, different variability modeling methods have been proposed. An important group of such methods are based on UML. These methods typically introduce profiles for specifying mandatory and optional elements, identifying dependencies between elements, and modeling variation points and possible variants. However, the assessment of these methods still lacks. In this work, we have done a first step towards evaluating the comprehension and utilization of variability issues in UMLbased models by suggesting a comparison framework which refers to different aspects of variability specification. Based on this framework, we chose a specific UML-based method - ADOM - and examined how advanced information systems students understood and utilized a model specified using this method. The results showed that the different means for specifying variability were understood and utilized only to a limited extent and that variation points were the least comprehensible variability specification means.
\end{abstract}

Keywords: variability management, software product line engineering, UML, ADOM.

\section{Introduction}

Software Product Line Engineering (SPLE) [25] deals with two main activities: domain engineering, during which a family of software products, termed a product line, is analyzed, designed, and implemented, and application engineering in which the particular applications and software products are customized and developed. Accordingly, Bachmann and Clements [3] distinguish between core assets and product artifacts: while core assets, also called domain artifacts, are built to be used by more than one product in the line, product artifacts are specific parts of the software products. In order to be reusable and suitable to a wide variety of products, the core assets have to specify, besides the commonality, the variability of the given product line. Variability is defined as the ability of a core asset to be efficiently 
extended, changed, customized, or configured for use in a particular product artifact [29]. Svahnberg et al. [31] claimed that "development of software product lines relies heavily on the use of variability to manage the differences between products", but "variability is not trivial to manage".

Variability modeling plays an important role in variability management [29]. Reviewing 97 papers that describe variability management methods in SPLE, reported from 1990s to 2007, Chen and Babar [6] conclude that the main corpus of methods focuses on variability modeling and utilizes feature models (33 works) or UML and its extensions (25 works) for this purpose. Feature-oriented methods, such as [20] and [22], support specifying core assets as sets of characteristics relevant to some stakeholders and the relationships and dependencies among them. Variability is specified in terms of mandatory vs. optional features, alternatives, OR features, 'require' and 'exclude' dependencies among features, feature groups, and composition rules. UML-based methods (e.g., [13], [26], [28], [32], and [34]) usually suggest profiles for handling variability-related issues, including specification of mandatory and optional elements, dependencies among elements, variation points, and possible variants [15]. Some UML-based methods suggest extending UML or representing variability aspects orthogonally to "regular" UML models of the product families, e.g., [14]. Other directions, such as applying Domain-Specific Languages (DSL), see for example [17] and [24], are also explored, but the number of works in each such category according to [6] is still very low.

Despite their amenability to be empirically evaluated, relatively minor attention is allocated for the empirical evaluation of SPLE methods in general and variability modeling issues in particular [6]. These studies highlight different aspects in SPLE, including product derivation [30], quality assurance [4, 9], and architecture process activities [1]. However, only one of them [4] refers to the comprehensibility of core assets. This work assesses the maintainability of feature models in terms of analyzability, changeability, and understandability, using a 7-point scale to gather the subjective opinions of the participants on the relevant characteristics. This work does not check the participants' performance in carrying out different domain or application engineering tasks.

As comprehensibility of core assets may affect the correctness of their utilization while creating valid product artifacts, we draw in this paper a general comparison framework for evaluating variability aspects in SPLE methods. This framework, which refers to both specification aids and their utilization during application engineering, is used for better understanding how to specify core assets while conducting domain engineering. According to this framework, the specification aids used in domain engineering are divided into two types: selection and extension. Selection variability refers to choosing elements from the core asset for a particular product artifact, while performing application engineering. It includes mandatory and optional elements specification, as well as dependencies specification. Extension variability, on the other hand, refers to locations in core assets at which variability may occur (variation point) and possible ways to realize this variability in order to create particular product artifacts (variants). Extension variability needs to answer different questions, such as: (1) Which variants can be selected at a certain variation point? (2) Can new variants be introduced for a particular product? (3) When will the variability be resolved? 
We concentrate here on variability modeling in UML-based methods, due to the popularity and the wide usage of their notation in software engineering in general, the ease of extending them to different product and product line aspects (including behavioral ones, design- and implementation-related aspects, and so on), and the minimal requirements for additional specification (as opposed, for example, to DSLs).

Based on the suggested framework, we reviewed different UML-based methods and selected the Application-based Domain Modeling (ADOM) method whose expressiveness in terms of variability specification includes most of the important concepts. We then carried out an empirical study on information systems students to examine how they understand a domain model (which is a type of core assets) specified using this method. We further checked how the domain model is utilized while creating specific models of applications (i.e., product artifacts in the line). Analyzing the study results, we claim that variability was understood and utilized only to a limited extent and that most of the problems were in understanding variation points and utilizing extension variability.

The remainder of this paper is organized as follows. Section 2 reviews existing methods for modeling variability using UML, whereas Section 3 introduces and exemplifies the ADOM method and its mapping to other UML-based variability modeling methods. Section 4 elaborates on the empirical study, discussing the research questions, the settings, the results, and the threats to validity. Finally, Section 5 concludes and refers to future research directions.

\section{Variability Modeling in UML-Based Methods}

Most UML-based methods in the field of SPLE define profiles to support the modeling of variability aspects, while some of them suggest modifications to the UML metamodel or specification of a "variability model" orthogonally to the UML models. Table 1 summarizes related work according to the way they specify selection and extension variability and the supported (UML) diagrams. As can be seen, most methods refer to both selection and extension variability. Mandatory (sometimes called kernel) and optional elements are usually specified using dedicated stereotypes, although these stereotypes sometimes refer only to variation points and variants and not to other elements in the core assets. Some works explicitly specify extension variability using both «variation point» and «variant» stereotypes, while others specify only one of these concepts and the other is implicitly specified from its relationships with the other concept. Several works explicitly refer to dependencies between elements in the form of «alternative_or», «alternative_XOR», «requires», and «mutux» stereotypes.

In order to evaluate the comprehension and utilization of variability aspects in UML-based methods and to identify factors that may affect these activities, we conducted an empirical study with a method, called Application-based DOmain Modeling (ADOM) [26, 27]. We chose this method over other UML-based methods since it explicitly refers to extension variability and in particular to the selection and the addition of variants in certain variation points, aspects which other UML-based methods tend to neglect. Furthermore, it enables explicit specification of both variation points and variants and it allows specifying ranges of multiplicity and not just mandatory and optional elements. 
Table 1. Comparison of Variability Modeling in UML-based Methods

\begin{tabular}{|c|c|c|c|}
\hline $\begin{array}{l}\text { Method } \\
\text { name }\end{array}$ & $\begin{array}{l}\text { Selection Variability } \\
\text { Specification }\end{array}$ & $\begin{array}{l}\text { Extension Variability } \\
\text { Specification }\end{array}$ & $\begin{array}{l}\text { Supported } \\
\text { diagrams }\end{array}$ \\
\hline RSEB [18] & & $\begin{array}{l}\text { Explicit specification of } \\
\text { variation points; Variants are } \\
\text { specializations of variation } \\
\text { points }\end{array}$ & $\begin{array}{l}\text { Use case and } \\
\text { class diagrams }\end{array}$ \\
\hline PLUS [13] & $\begin{array}{l}\text { Distinction between «kernel» } \\
\text { (mandatory) and «optional» } \\
\text { elements }\end{array}$ & $\begin{array}{l}\text { Explicit specification of variants } \\
\text { only («variant») }\end{array}$ & All diagrams \\
\hline $\begin{array}{l}\text { Halmans \& } \\
\text { Pohl [14] }\end{array}$ & $\begin{array}{l}\text { Specification of mandatory and } \\
\text { optional variation points via black } \\
\text { and white triangles, respectively }\end{array}$ & $\begin{array}{l}\text { Explicit specification of variants } \\
\text { («variant»); Explicit } \\
\text { relationships between variants } \\
\text { and variation points via } \\
\text { «include» dependencies }\end{array}$ & $\begin{array}{l}\text { Use case } \\
\text { diagrams }\end{array}$ \\
\hline $\begin{array}{l}\text { Ziadi et al. } \\
\text { [34] }\end{array}$ & $\begin{array}{l}\text { Possibility to classify elements as } \\
\text { «optional» }\end{array}$ & $\begin{array}{l}\text { Explicit specification of both } \\
\text { variation points («variation») } \\
\text { and variants («variant»); } \\
\text { Variants inherit variation points, } \\
\text { which are modeled as abstract } \\
\text { classes }\end{array}$ & $\begin{array}{l}\text { Class and } \\
\text { sequence } \\
\text { diagrams }\end{array}$ \\
\hline $\begin{array}{l}\text { Alves de } \\
\text { Oliveira et } \\
\text { al. [1] }\end{array}$ & $\begin{array}{l}\text { Classification of variants as } \\
\text { «mandatory», «optional», } \\
\text { «alternative_or», } \\
\text { «alternative_XOR» for a certain } \\
\text { variation point; Dependencies } \\
\text { between variants are marked by } \\
\text { «requires» and «mutux» }\end{array}$ & $\begin{array}{l}\text { Explicit specification of } \\
\text { variation points }\end{array}$ & $\begin{array}{l}\text { Use case, } \\
\text { class, and } \\
\text { component } \\
\text { diagrams }\end{array}$ \\
\hline $\begin{array}{l}\text { Robak et } \\
\text { al. [28] }\end{array}$ & & $\begin{array}{l}\text { Explicit specification of } \\
\text { variants; Variation points are } \\
\text { specified as branches in activity } \\
\text { diagrams or components in } \\
\text { component diagrams }\end{array}$ & $\begin{array}{l}\text { Component } \\
\text { and activity } \\
\text { diagrams }\end{array}$ \\
\hline SPLIT [8] & $\begin{array}{l}\text { The existence attribute indicates } \\
\text { whether a variation point is } \\
\text { optional or mandatory }\end{array}$ & $\begin{array}{l}\text { Explicit specification of } \\
\text { variation points; Relationships } \\
\text { between variants and their } \\
\text { variation points are classified } \\
\text { according to the variability } \\
\text { mechanisms («insert», «extend», } \\
\text { or «parameterize») }\end{array}$ & All diagrams \\
\hline VPM [32] & $\begin{array}{l}\text { Mandatory variation points are } \\
\text { specified as 'm', while optional } \\
\text { variation points are specified as 'o' }\end{array}$ & $\begin{array}{l}\text { Explicit specification of } \\
\text { variants; Variation points are } \\
\text { visualized and categorized into } \\
\text { four types: Parameterization, } \\
\text { Information Hiding, Inheritance, } \\
\text { and Callback }\end{array}$ & All diagrams \\
\hline $\begin{array}{l}\text { ADOM } \\
{[26,27]}\end{array}$ & $\begin{array}{l}\text { Associating the stereotype } \\
\text { «multiplicity min=m max=n»; } \\
\text { Dependencies can be marked as } \\
\text { «requires» or «excludes» }\end{array}$ & $\begin{array}{l}\text { Explicit specification of both } \\
\text { variation points («variation } \\
\text { point») and variants («variant»); } \\
\text { Variants inherit variation points; } \\
\text { Variation points may constrain } \\
\text { the selection and addition of } \\
\text { variants }\end{array}$ & All diagrams \\
\hline
\end{tabular}




\section{The ADOM Method}

The Application-based DOmain Modeling (ADOM) method is based on a profile with five stereotypes, presented in Figure 1: «multiplicity», «variation point», «variant», «requires», and «excludes». The «multiplicity» stereotype, which is elaborated in [26], is used for specifying the range of elements in a product artifact that can be classified as the same element in the core asset. Two tagged values, min and max, are used for defining the lowest and upper-most boundaries of that range. For clarity purposes, four commonly used multiplicity groups are defined on top of this stereotype: «optional many», where $\min =0$ and $\max =\infty$, «optional single», where $\min =0$ and $\max =1$, «mandatory many», where $\min =1$ and $\max =\infty$, and «mandatory single», where $\min =\max =1$. Nevertheless, any multiplicity interval constraint can be specified using the general stereotype «multiplicity $\min =\mathrm{m}_{1} \max =\mathrm{m}_{2} »$.
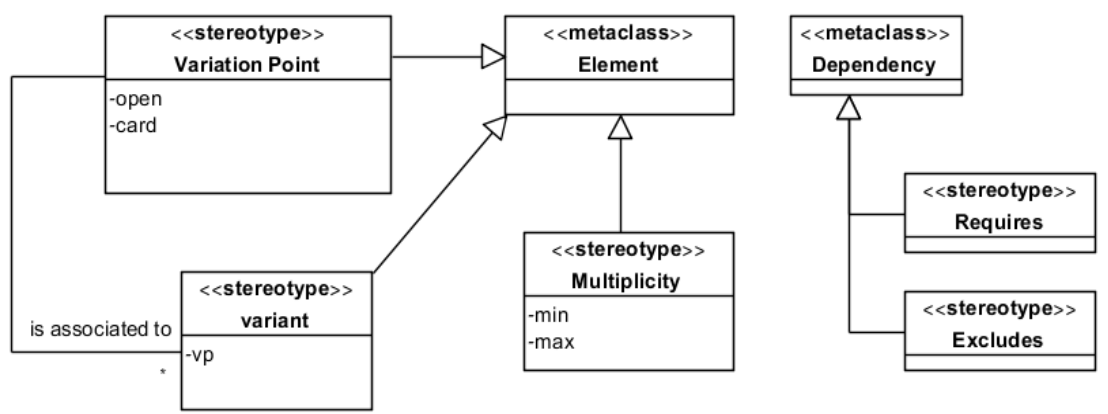

Fig. 1. ADOM's profile

Each element in the core asset may be defined as a variation point. This is done using the stereotype «variation_point», in addition to the «multiplicity» stereotype. A «variation_point»stereotype has the following tagged values: (1) open, specifying whether the variation point is open or closed, i.e., whether specific variants that are not specified in the core asset can be added at this point in a particular product or not, and (2) card(inality), indicating the number of variant types need to be chosen for this variation point; common cardinalities are '1..1' (XOR), '1..*' (OR), '0..1' (optional $\mathrm{XOR}$ ), and '0..*' (optional OR). Note that there are differences between the «multiplicity» stereotype and the cardinality tagged values. A variation point, for example, can be optional while its cardinality specification is mandatory (e.g., '1..*'), indicating that this variation point may not be included in a particular product, but if it is, then at least one of its variants (as specified in the core asset) have to be selected. Similarly, an open variation point can be mandatory while its cardinality specification is optional (e.g., '0..*'), indicating that this variation point has to be included in a particular product, but possibly use particular, product-specific variants (which are not specified in the core asset). 
Each variant is specified using the «variant» stereotype, in addition to the «multiplicity» stereotype. A variation point and its variants should be of the same type (e.g., classes, attributes, associations, etc.). In the literature the relationships between a variation point and its variants may take various forms, e.g., inheritance and dependency [7]. Since variation points may specify structural and behavioral aspects that are relevant to all their variants, ADOM supports specifying the relationship between a variant and the relevant variation point via inheritance relationships. When not applicable, i.e., for variation points and variants that are not classifiers, such as attributes, operations, and combined fragments, the relationships between variants and variation points are specified using a tagged value, $v p$, associated to the «variant» stereotype; $v p$ specifies the corresponding variation point. Note that the same element in the core asset can be stereotyped by both «variation_point» and «variant», enabling specification of hierarchies of variants.

Finally, two stereotypes are defined for determining dependencies between elements (and possibly between variation points and variants): «requires» and «excludes». A «requires» B, where A and B are two (optional) elements, implies that if A appears in a particular product artifact, then B should appear too. Similarly, A «excludes» $\mathrm{B}$ implies that if $\mathrm{A}$ is included in a particular product artifact, then $\mathrm{B}$ should not.

The suggested profile includes in addition a set of rules that specify the allowed combination of stereotypes. For example, the cardinality constraints of a variation point should be feasible in the context of the multiplicity constraints of its variants. However, due to space limitations, the entire set of rules is not presented here.

Figure 2 is a part of a Check-In/Check-Out (CICO) product line specification. The main purpose of this product line is developing applications or software products for checking in and out items. Examples of products in this line are hotel reservation systems, libraries, renting agencies, and version control services. Items that can be checked out have unique identifiers, as well as attributes specifying their statuses, (general) details, check-in details, and check-out details. They are primarily divided into virtual and physical items. Based on this model, handling fees differ according to the item type: physical items must have delay fees handling, whereas damage and lost fee calculations are optional. They further have location details. Virtual items, on the other hand, typically have no location details and no delay, lost, and damage fees. They may need to handle fees when the specified loan policy is violated. According to the associated tagged values of the variation point (open is true and cardinality is optional OR, i.e., '0..*'), a particular application model may include items which are neither physical nor virtual.

In a hotel reservation application, for example, the hotel rooms can be classified as physical items, whereas the different services provided by the hotel can be considered as virtual items. We could specify in the domain model an «excludes» dependency between Physical Item and Virtual Item, indicating that each product in the line may handle either physical or virtual items (but not both), but we did not do that in order to allow creation of CICO software products that handle both physical and virtual items, such as hotel reservation applications.

To check the expressiveness of ADOM with respect to the other reviewed works, we mapped the different stereotypes of ADOM to those of the reviewed methods. Table 2 presents this mapping. As can be seen, the important concepts are explicitly supported by ADOM. 


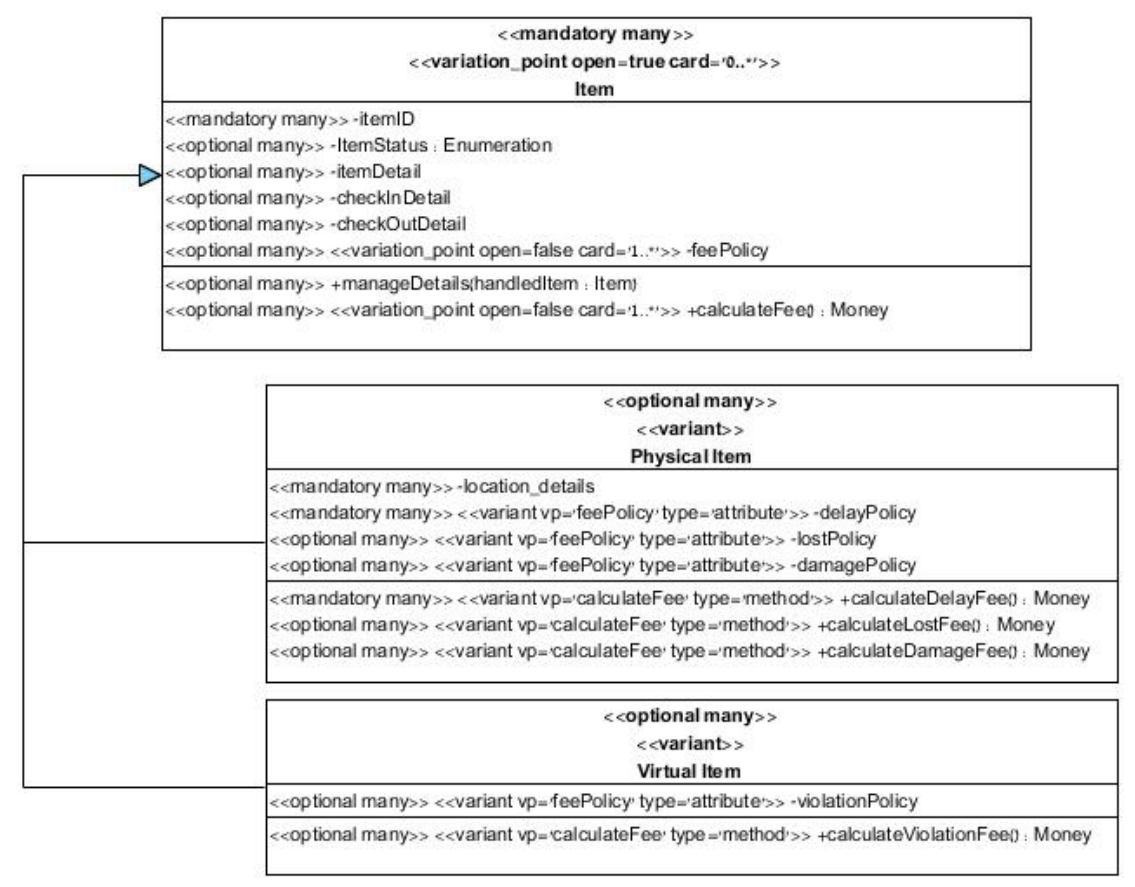

Fig. 2. Specification of Item variation point in ADOM

Table 2. Mapping between ADOM and other UML-based methods

\begin{tabular}{||l|l||}
\hline \hline Stereotype in ADOM & Corresponding stereotypes in the related works \\
\hline \hline «mandatory single», «mandatory many» & «kernel» [13], «mandatory» [1] \\
\hline «optional single», «optional many» & «optional» [1,13,34] \\
\hline «variation point» & «variation point» [1, 8], «variation» [34], «virtual» [34] \\
\hline «variant» & «variant» [13, 14, 18, 32, 34] \\
\hline Cardinality='1..*' & «alternative_or» [1] \\
\hline Cardinality='1..1 & «alternative_XOR» [1], «insert» [8] \\
\hline «requires» & «requires» [1] \\
\hline «excludes» & «mutux» $[1]$ \\
\hline $\begin{array}{l}\text { Inheritance between variation point and } \\
\text { variant }\end{array}$ & «extend» [8], «include» [14] \\
\hline \hline
\end{tabular}

\section{Comprehension and Utilization of Variability Aspects in ADOM}

In order to examine the comprehension and utilization of variability aspects in ADOM, we conducted an empirical study, which aimed at checking the following two research questions:

Question \#1 (comprehension): Is selection and extension variability as specified in ADOM's models well understood to software modelers and to what extent? 
Question \#2 (utilization): Is selection and extension variability as specified in ADOM's models well utilized by software modelers and to what extent? Here we examined two common utilizations of core assets, namely validation of a particular product with respect to the relevant core asset and guidance of particular product creation. Both guidance and validation activities are part of application engineering and are conducted while designing particular product artifacts.

The settings, results, and threats to validity of this study are reported below.

\subsection{Study Settings}

Since it is very difficult to find suitable subjects for such empirical studies - subjects who know the required material and who are willing to devote their time - we conducted the study with 15 advanced (last year) undergraduate and graduate students in an Information Systems program at the University of Haifa, Israel, who took a seminar course on domain engineering during the winter semester of the academic year 2009-2010. All these students had previous knowledge in software systems modeling and specification (this was their third course on this subject), as well as initial experience in industrial projects. Thus, they can be considered comparable to junior software modelers. During the course, the students studied various domain engineering techniques, focusing on the ADOM method and its ability to specify, guide, and validate variability. They further got homework exercises in which they had to model in groups of four students a domain model and four application models in the same domain or product line, using that domain model. Besides this, the students were not explicitly trained towards the experiment's tasks.

The study took place towards the end of the course as a class assignment. This assignment was worth up to 10 points of the students' grades in the course. The students got an ADOM model of a Check-in/Check-out product line. We chose this example since we believe that the students are familiar with different applications in this domain, such as library, car rental, and hotel reservation systems. This way the terminology used for specifying the core asset was not strange or unfamiliar to them. The model included 3 use case diagrams, 3 class diagrams, and 3 sequence diagrams. In each diagram type, one diagram was considered main (or top level), while the others elaborated the different variants of certain variation points. Overall, seven variation points were specified. Two experts checked the models before the experiment took place and especially their correctness and the ability to answer the questions according to the models. The students had to answer questions that referred to comprehension, validation, and guidance of the given model, as described in the next sub-sections ${ }^{1}$.

\subsection{Comprehension Questions and Results}

The first task, which referred to research question \#1, included 14 true/false questions regarding the given $\mathrm{CICO}$ model. Examples of questions in this part are:

\footnotetext{
1 The complete questionnaire can be found at http://mis.hevra.haifa.ac.il/ iris/research/SPLEeval/
} 
A product in the line may have the possibility to reserve items. In case the product supports this functionality, both loaners and workers should be able to perform it. (a use case diagram related question)

A loaner may handle his/her lending by performing and modifying it. The product should enable recording these operations, and in particular the times in which they occur. (a class diagram related question)

A product in the line may get the item details in a check out scenario by finding this item through the collections it belongs to. (a sequence diagram related question)

For this task, the answers were checked according to a pre-defined solution. For each question we analyzed the answer correctness by examining both the final answer (true/false) and the explanation. An incorrect answer or explanation scored 0, a fully correct answer or explanation scored 1 point, and a partially correct explanation scored 0.5 . We grouped the various questions according to the variability types they referred to. Table 3 summarizes the average scores achieved by the students in each category, divided according to the diagram types. For enabling comparison, all scores were converted to percentages that represent the success or correctness rate. As can be seen, the explanation scores were lower than the answer scores in the various question categories. We believe that the reason for that is the difficulty of the students to explicitly point out the reasons for their answers and to justify them.

When analyzing the selection variability, we noticed that the students performance in the use case diagrams were very low. Since this type of diagram is relatively simple, we deeply analyzed the questions in this category and figured out that most of them referred to a model segment rather than to particular elements. The students had problems to find the right answers since they had to gather information from different sources (the elements that composed the segment).

Table 3. Results of the first part (comprehension)

\begin{tabular}{|c|c|c|c|c|}
\hline \multicolumn{2}{|r|}{ Method } & Answer & Explanation & Comments \\
\hline \multirow{4}{*}{ 兽 } & Use Case & $40.0 \%$ & $40.0 \%$ & $\begin{array}{l}\text { The questions in this category referred to segments in } \\
\text { the UC diagrams and not to individual elements. }\end{array}$ \\
\hline & Class & $76.7 \%$ & $53.3 \%$ & \\
\hline & Sequence & $80.0 \%$ & $63.3 \%$ & \\
\hline & Overall & $62.7 \%$ & $50.0 \%$ & \\
\hline \multirow{4}{*}{ 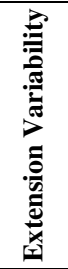 } & Use Case & $51.1 \%$ & $44.4 \%$ & $\begin{array}{l}\text { All questions in this category referred to variation points } \\
\text { and how to select particular variants. }\end{array}$ \\
\hline & Class & $55.6 \%$ & $32.2 \%$ & $\begin{array}{l}\text { All questions in this category referred to particular } \\
\text { variants rather than to variation points. }\end{array}$ \\
\hline & Sequence & $46.7 \%$ & $28.9 \%$ & $\begin{array}{l}\text { The questions in this category referred to both variants } \\
\text { and variation points. }\end{array}$ \\
\hline & Overall & $51.1 \%$ & $35.2 \%$ & $\begin{array}{l}\text { Overall, the performance of variants-related questions } \\
\text { was better than that of variation points-related questions. }\end{array}$ \\
\hline
\end{tabular}

When analyzing the extension variability results, we found out that the performance in all diagram types was relatively low, 46\%-56\%. Trying to reach a deeper insight, we separately examined the comprehension of variation points and the 
comprehension of variants and we found out that the performance in variant-related questions was better than the performance in variation point-related questions $(56 \%$ vs. $45 \%$ on the average). Our conjecture regarding this observation is that variation points are more abstract, usually refer to several elements (variants) and include information regarding the way to realize the variability. Thus, their specification may be more difficult to understand than that of variants, which are more concrete and focus on particular elements.

\subsection{Validation Questions and Results}

The second task, which partially referred to research question \#2, included a model of a hotel reservation application, which is in the scope of the Check-In/Check-Out product line. Following the domain and application models the students were requested to identify places where the hotel reservation model violates the product line constraints. Due to difficulties in defining and understanding the semantics of sequence diagrams [33], we chose to concentrate in this part on functional and structural aspects only. Thus, the hotel reservation model consisted of a use case diagram and a class diagram. The violations referred to both selection and extension variability. Differently from the first task, this part was open and the students were not notified regarding the numbers of violations or their distribution.

For checking this task, we prepared a list of 9 mistakes (or inaccuracies) in the hotel reservation application, according to the corresponding domain model. As we had to analyze the relevance of the mistakes found by the students with respect to our predefined list, we measured the performance in this part in terms of precision, recall, and F-measure [23], which are standard metrics for measuring the relevance of retrieved items (mistakes or inaccuracies in our case). Precision measures the fraction of items in the answer that are correct, while recall measures the fraction of expected items that are in the answer. Since the two above metrics measure different concepts, we used F-measure, which is a derived measure defined as the harmonic mean of precision and recall [23]:

$$
\mathrm{F}-\text { measure }=\frac{2 * \text { precision } * \text { recall }}{\text { precision }+ \text { recall }} .
$$

Table 4 summarizes these measurements according to the two variability types, selection and extension, and the two diagram types. As can be clearly seen through the F-measurement, the results are poor, especially when looking at the low values of recall (less than 20\% in the overall). However, errors that referred to selection variability were much easier to find than errors that referred to extension variability. Although the recall in the selection variability category was quite similar in use case and class diagrams ( $\sim 27 \%$ and $\sim 36 \%$, respectively), the precision was quite different: the precision of finding errors in use case diagrams was lower than the precision of finding errors in class diagrams. One possible explanation to this observation is that the students were more familiar and experienced with class diagrams (a case that is also reported in [11]). Another explanation is that the use case diagram referred to two basic operations: check-out, i.e., borrowing an item, which is called check-in in the hotel application, and check-in, i.e., returning an item, which is termed check-out in the hotel application. The swapping of these terms in the particular application caused a lot of difficulties to the students. 
Table 4. Results of the second task (validation)

\begin{tabular}{|c|c|c|c|c|}
\hline \multicolumn{2}{|c|}{ Method } & Precision & Recall & F-measure \\
\hline \multirow{3}{*}{$\begin{array}{l}\text { Selection } \\
\text { Variability }\end{array}$} & $\mathbf{U C}$ & 29.86 & 26.67 & 28.17 \\
\hline & Class & 58.93 & 35.56 & 44.35 \\
\hline & Overall & 45.92 & 32.00 & 37.72 \\
\hline \multirow{3}{*}{$\begin{array}{l}\text { Extension } \\
\text { Variability }\end{array}$} & UC & 100.00 & 3.33 & 6.45 \\
\hline & Class & 100.00 & 3.33 & 6.45 \\
\hline & Overall & 100.00 & 3.33 & 6.45 \\
\hline \multicolumn{2}{|l|}{ Overall } & 47.58 & 19.26 & 27.42 \\
\hline
\end{tabular}

In the extension variability category, only one student found one error in the use case diagram and another student found a single error in the class diagram. In general, we believe that the reason for this result is the fact that the mistakes and inaccuracies were not obvious and spanned within different model elements.

Analyzing the students' explanations in this part, we found three sources of problems. First, the students had difficulties in mapping the elements of the hotel reservation application to the CICO elements. As this mapping may reveal anchors for validating particular product artifacts with respect to core assets, these difficulties also prevent the students from correctly identifying problems that relate to selection variability. Second, checking each element in the application against its corresponding core asset's element, which may appear in several diagrams (e.g., in the case of a variation point), is a difficult and exhaustive task that may yield inconsistencies in the provided answers. This problem, which probably affected the low recall in all categories and especially in the extension variability category, supports the need for well established processes, techniques, and tools for validating particular product artifacts against their relevant core assets. Finally, the openness of this task caused the students to find problems that were not actually mistakes, while trying to generate complete lists of problems, partially explaining the low precision in the selection variability category.

\subsection{Guidance Questions and Results}

Finally, in the third part, which also referred to research question \#2, the students got a list of requirements for a library application, which also belongs to the Check In/ Check Out product line. Based on the domain model and the library requirements, the students were asked to develop a specific model for the library application. Examples of requirements in this part are:

Some of the copies can be borrowed online, without visiting the library and getting the actual copies. However, a loaner can get these copies also physically.

Online borrowers may not be members of the library. In this case, they have to pay for each online borrowing, before the copy is borrowed. Members can pay for their online borrowing when the due date of the borrowing arrives or when the online copy is expired.

If the book or journal copy has been damaged during the borrowing, the librarian will ask the system to calculate the damage charges for the specific book or journal. For each book or journal, there are 3 damage fees: minor damage fees, medium damage fees, and major damage fees. 
In this part, we examined how the aids for specifying selection and extension variability were utilized for guiding the creation of models for the library application. As different models could be provided for the same set of requirements, we graded the models correctness and completeness with respect to the library requirements.

Analyzing the results, we found out that the specification of selection variability guides the students to a large extent. Thus, in the following we chose to concentrate on the extension variability specification. In particular, we separately analyzed how variation points and variants are utilized for guiding application development. Table 5 summarizes the results achieved in the third part of the study with respect to the extension variability. The different numbers indicate the numbers of students (out of 15) correctly/wrongly specified each variation point and its related variants. Variation point specification refers to the selection and addition of the different variants (as expressed in the open and cardinality tagged values), whereas variant specification refers to the structure of the relevant variants as specified in the domain model or inherited from the variation point specification. All the variation points were required for completely specifying the library application.

We further examined the correlation between the success of utilizing a variation point and the success of utilizing its variant(s) using the Phi coefficient which is suitable for analyzing the correlation between two binary variables [12]. As can be seen, in most cases there is a correlation between the success of utilizing variation points and their associated variants. Two exceptions to this conclusion are the loaner and the item variation points. In the loaner case, which appeared in the use case diagrams, the variation point specified a cardinality of exactly 3 (i.e., a loaner can be either physical or virtual, either member or occasional loaner, and either cooperation or private). Almost all students (14 out of 15) did not enforce this constraint. However, all of them succeeded in modeling the different loaners (variants) and their relations to use cases. Regarding items, there was confusion between the actual items (copies) and their types (e.g., books). Nevertheless, when modeling a particular variant, the students followed all the provided guidance.

Table 5. Results of the third task (guidance)

\begin{tabular}{||l|l|l|l||c|c|c||}
\hline$\#$ & $\begin{array}{l}\text { Variation point } \\
\text { (VP) name }\end{array}$ & $\begin{array}{l}\text { Diagram } \\
\text { type }\end{array}$ & $\begin{array}{l}\text { Element } \\
\text { type }\end{array}$ & $\begin{array}{c}\text { VP } \\
\text { Utilization }\end{array}$ & $\begin{array}{c}\text { Variant } \\
\text { Utilization }\end{array}$ & $\begin{array}{c}\text { Correlation } \\
\text { (Phi) }\end{array}$ \\
\hline \hline 1 & Loaner & Use case & Actor & $1 / 15$ & $15 / 15$ & $\begin{array}{c}-1.00 \\
\text { (est.sig=.157) }\end{array}$ \\
\hline 2 & Check-In Item & Use case & Use case & $13 / 15$ & $13 / 15$ & $1^{*}$ \\
\hline 3 & Item & Class & Class & $4 / 15$ & $15 / 15$ & $\begin{array}{c}-1.00 \\
\text { (est.sig }=.157)\end{array}$ \\
\hline 4 & Fee & Class & Attribute & $1 / 15$ & $1 / 15$ & $1^{*}$ \\
\hline 5 & calculateFee & Class & Operation & $4 / 15$ & $4 / 15$ & $1^{*}$ \\
\hline 6 & Handle & Class & Association & $15 / 15$ & $15 / 15$ & $1^{*}$ \\
\hline 7 & getItemDetails & Sequence & $\begin{array}{l}\text { Comb. } \\
\text { Fragment }\end{array}$ & $9 / 13$ & $9 / 13$ & $1^{*}$ \\
\hline \hline
\end{tabular}

* The Phi coefficient cannot be computed due to exact match.

** Two students completely ignored this variation point and thus were omitted from the statistical analysis of the utilization correlation. 
Another observation that can be made on the results presented in Table 5 is that the guidance provided by variation points was less considered than the guidance provided by the variants. With respect to the variation point guidance, only 2 variation points were used by most of the students (\#2 and \#6 in the table), 1 variation point was used by many students (\#7) and the other variation points were used to a very limited extent (\#1, \#3, \#4, and \#5). With respect to the variant guidance, most (5) variants were used by most of the students (\#1, \#2, \#3, \#6, and \#7), while the other 2 variants were used to a very limited extent (\#4 and \#5). It can also be seen that low level elements, such as attributes and operations, were less handled with regard to both variation point and variant specifications (\#4, \#5, and \#7). A possible reason for this may be that the students concentrated on main, top level concepts and the relationships between them, neglecting low level details.

\subsection{Threats to Validity}

In our study, we found out that variability is understood and utilized to a limited extent and that extension variability is more difficult to understand and utilized. Nevertheless, these results should be discussed under the following validity threats that are explained below, along with the actions we made to overcome these threats.

The first threat concerns the number and the type of employed subjects. We carried out the study with only 15 students, due to the difficulty to find suitable subjects who are willing to devote their time. However, these students got very good grades in software systems modeling and domain engineering throughout the entire course (an average of 91). Furthermore, they were trained with the suggested profile and motivated (by 10 points of their final grades) to perform the requested tasks well. Kitchenham et al. [21] argue that using students as subjects instead of software engineers is not a major issue, as long as the research questions are not specifically focused on experts. In our case, the expected users of the method are software developers, or more accurately modelers, who are not necessarily familiar with the given domain. Thus, the selected students may represent the expected users. Only further studies may confirm or disconfirm whether our results can be generalized to more experienced subjects (e.g., software developers and modelers in industry).

The second threat we need to refer to is the simple tasks and models used in the study (this was done in order to adjust the tasks to the capabilities of the subjects within the time frame). On this issue, we can say that the models were carefully built referring to different variability-related challenges. However, we aim at keeping the models simple, yet realistic. Note that despite the small models, essential problems were encountered, making it interesting to check how things would result with real systems and large domains.

Finally, the study was carried out with a specific method, ADOM. Although this method was selected after a careful examination of different UML-based methods (see the resultant mapping in Table 2), comparative analysis needs to be done in order to check the comprehension and utilization capabilities of different methods in this category. Such analysis can be done by defining a set of criteria, similar to the ones listed, for example, in [10] and [16], and examining how the different methods satisfy these criteria. Complementarily, this analysis can use comparative empirical evaluation techniques, involving several UML-based methods. 


\section{$5 \quad$ Summary and Future Work}

Variability specification is important as it may help create valid applications in certain domains. In this paper, we provide some empirical evidence to the difficulties in comprehending and utilizing variability specified in domain models and potential sources for these difficulties. We found out that variability was understood and utilized to a limited extent. The enforcement of variability-related constraints, especially those that refer to extension variability, and the creation of specific models in the domain were very difficult tasks. We found that it was especially difficult to utilize a domain model for validating a specific application model in the domain. Furthermore, the guidance of the domain model with respect to variation points was very limited when creating a new application model. In general, we found out that variation points were less understood and utilized than selection variability and variants. These findings call for developing explicit and focused methods and tools that will help comprehend variability aspects, as well as the allowed and forbidden options of applications, in certain domains.

In the future, we will replicate the empirical study on larger classes of trained domain engineering students and software modelers and develop case studies of larger domains. Moreover, we intend to revise the tasks and use them for comparing additional variability modeling methods. These can be done in the era of UML-based methods, but other alternatives also exist. For example, a possible solution may be the adoption of a variability language such as the emerging standard of CVL [17] or the utilization of feature-oriented approaches as model composition [19].

We also plan to analyze the results with respect to cognitive theories, such as those presented in [5], in order to gain further understanding on the ways according to which variability modeling methods should be designed.

\section{References}

1. Ahmed, F., Capretz, L.F.: The software product line architecture: An empirical investigation of key process activities. Information and Software Technology 50, 10981113 (2008)

2. Alves de Oliveira, E., Gimenes, I.M.S., Huzita, E.H.M., Maldonado, J.C.: A Variability Management Process for Software Products Lines. In: Centre, I.B.M. (ed.) for Advanced Studies Conference Archive, Proceedings of the 2005 Conference of the Centre for Advanced Studies on Collaborative Research Table of Contents, Toranto, Ontario, Canada, pp. 225-241 (2005)

3. Bachmann, F., Clements, P.C.: Variability in Software Product Lines. Technical Report CMU/SEI-2005-TR-012 (2005),

http: / / www . sei.cmu . edu/library/abstracts/reports / 05 tr012 . cfm

4. Bagheri, E., Dasevic, G.: Assessing the Maintainability of Software Product Line Feature Models using Structural Metrics. Software Quality Journal (2011), doi:10.1007/s11219010-9127-2

5. Bajaj, A., Rockwell, S.: COGEVAL: Applying Cognitive Theories to Evaluate Conceptual Models. Advanced Topics in Database Research (4), 255-282 (2005) 
6. Chen, L., Babar, M.A.: A systematic review of evaluation of variability management approaches in software product lines. Information and Software Technology 53, 344-362 (2011)

7. Clotet, R., Dhungana, D., Franch, X., Grunbacher, P., Lopez, L., Marco, J., Seyff, N.: Dealing with Changes in Service-Oriented Computing Through Integrated Goal and Variability Modelling. In: Proceeding of the Second International Workshop on Variability Modelling of Software-intensive Systems (VaMoS 2008), pp. 43-52 (2008)

8. Coriat, M., Jourdan, J., Fabien, B.: The SPLIT method: building product lines for software-intensive systems. In: Proceedings of the First Conference on Software Product Lines: Experience and Research Directions: Experience and Research Directions, pp. 147$166(2000)$

9. Denger, C., Kolb, R.: Testing and Inspecting Reusable Product Line Components: First Empirical Results. In: Proceedings of the 2006 ACM/IEEE International Symposium on Empirical Software Engineering, pp. 184-193. ACM (2006)

10. Djebbi, O., Salinesi, C.: Criteria for Comparing Requirements Variability Modeling Notations for Product Lines. In: The Fourth International Workshop on Comparative Evaluation in Requirements Engineering (CERE 2006), in Conjunction with RE 2006 (2006)

11. Dobing, B., Parsons, J.: How UML is used. Communication of the ACM 49(5), 109-113 (2006)

12. Edwards, A.L.: The Phi Coefficient. In: An Introduction to Linear Regression and Correlation, pp. 68-72. W. H. Freeman, San Francisco (1976)

13. Gomaa, H.: Designing Software Product Lines with UML: From Use Cases to PatternBased Software Architectures. Addison-Wesley Professional (2004)

14. Halmans, G., Pohl, K.: Communicating the Variability of a Software-Product Family to Customers. Software and Systems Modeling 2(1), 15-36 (2003)

15. Halmans, G., Pohl, K., Sikora, E.: Documenting Application-Specific Adaptations in Software Product Line Engineering. In: Bellahsène, Z., Léonard, M. (eds.) CAiSE 2008. LNCS, vol. 5074, pp. 109-123. Springer, Heidelberg (2008)

16. Haugen, Ø., Møller-Pedersen, B., Oldevik, J.: Comparison of System Family Modeling Approaches. In: Obbink, H., Pohl, K. (eds.) SPLC 2005. LNCS, vol. 3714, pp. 102-112. Springer, Heidelberg (2005)

17. Haugen, Ø., Møller-Pedersen, B., Oldevik, J., Olsen, G.K., Svendsen, A.: Standardized Variability to Domain Specific Languages. In: Proceedings of the 12th International Conference on Software Product Line, pp. 139-148 (2008)

18. Jacobson, I., Griss, M., Jonsson, P.: Software Reuse-Architecture, Process and Organization for Business Success. ACM Press, New York (1997)

19. Jayaraman, P., Whittle, J., Elkhodary, A.M., Gomaa, H.: Model Composition in Product Lines and Feature Interaction Detection Using Critical Pair Analysis. In: Engels, G., Opdyke, B., Schmidt, D.C., Weil, F. (eds.) MODELS 2007. LNCS, vol. 4735, pp. 151165. Springer, Heidelberg (2007)

20. Kang, K., Cohen, S., Hess, J., Novak, W., Peterson, A.: Feature-Oriented Domain Analysis (FODA) Feasibility Study. Technical Report CMU/SEI- 90-TR-21, Software Engineering Institute, Carnegie Mellon University (1990)

21. Kitchenham, B.A., Lawrence, S., Lesley, P., Pickard, M., Jones, P.W., Hoaglin, D.C., Emam, K.E.: Preliminary Guidelines for Empirical Research. IEEE Transactions on Software Engineering 28(8), 721-734 (2002) 
22. Kyo, C.K., Sajoong, K., Jaejoon, L., Kijoo, K., Euiseob, S., Moonhang, H.: FORM: A feature oriented reuse method with domain - specific reference architectures. Annals of Software Engineering 5(1), 143-168 (1998)

23. Manning, C.D., Raghavan, P., Schütze, H.: Introduction to Information Retrieval. Cambridge University Press (2008)

24. Mernik, M., Heering, J., Sloane, A.M.: When and How to Develop Domain-Specific Languages. ACM Computing Surveys (CSUR) 37(4), 316-344 (2005)

25. Pohl, K., Böckle, G., van der Linden, F.: Software Product Line Engineering: Foundations, Principles, and Techniques. Springer, Heidelberg (2005)

26. Reinhartz-Berger, I., Sturm, A.: Utilizing Domain Models for Application Design and Validation. Information and Software Technology 51(8), 1275-1289 (2009)

27. Reinhartz-Berger, I., Tsoury, A.: Experimenting with the Comprehension of FeatureOriented and UML-Based Core Assets. In: Halpin, T., Nurcan, S., Krogstie, J., Soffer, P., Proper, E., Schmidt, R., Bider, I. (eds.) BPMDS 2011 and EMMSAD 2011. LNBIP, vol. 81, pp. 468-482. Springer, Heidelberg (2011)

28. Robak, S., Franczyk, B., Politowicz, K.: Extending the UML for modeling variability for system families. International Journal of Applied Mathematics and Computer Science 12(2), 285-298 (2002)

29. Sinnema, M., Deelstraa, S.: Classifying Variability Modeling Techniques. Information and Software Technology 49(7), 717-739 (2007)

30. Sinnema, M., Deelstra, S.: Industrial validation of COVAMOF. Journal of Systems and Software 81(4), 584-600 (2008)

31. Svahnberg, M., Van Gurp, J., Bosch, J.: A Taxonomy of Variability Realization Techniques. Software - Practice \& Experience 35(8), 705-754 (2005)

32. Webber, D., Gomaa, H.: Modeling variability in software product lines with variation point model. Science of Computer Programming 53, 305-331 (2004)

33. Xiaoshan, L., Zhiming, L., Jifeng, H.: A formal semantics of UML sequence diagram. In: Australian Software Engineering Conference, pp. 168-177 (2004)

34. Ziadi, T., Hélouët, L., Jézéquel, J.M.: Towards a UML Profile for Software Product Lines. In: van der Linden, F.J. (ed.) PFE 2003. LNCS, vol. 3014, pp. 129-139. Springer, Heidelberg (2004) 